\title{
DESENVOLVIMENTO E CARACTERIZAÇÃO DE NANOCÁPSULAS DE POLI (L-LACTÍDEO) CONTENDO BENZOCAÍNA
}

Nathalie Ferreira Silva de Melo, Renato Grillo, André Henrique Rosa e Leonardo Fernandes Fraceto*

Departamento de Engenharia Ambiental, Universidade Estadual Paulista Júlio de Mesquita Filho, Av. Três de Março, 511, 18087-180 Sorocaba - SP, Brasil

Newton Luiz Dias Filho

Departamento de Física e Química, Universidade Estadual Paulista Júlio de Mesquita Filho, Campus Ilha Solteira, 15385-000

Iha Solteira - SP, Brasil

Eneida de Paula

Departamento de Bioquímica, Universidade Estadual de Campinas, Cidade Universitária “Zeferino Vaz", 13083-970 Campinas - SP, Brasil

Daniele Ribeiro de Araújo

Centro de Ciências Naturais e Humanas, Universidade Federal do ABC, Rua Santa Adélia, 166, 09210-400 Santo André - SP, Brasil

Recebido em 12/12/08; aceito em 23/6/09; publicado na web em 13/11/09

DEVELOPMENT AND CHARACTERIZATION OF POLI (L-LACTIDE) NANOCAPSULES CONTAINING BENZOCAINE. In this paper we describe the preparation poly (L-lactide) (PLA) nanocapsules as a drug delivery system for the local anesthetic benzocaine. The characterization and in vitro release properties of the system were investigated. The characterization results showed a polydispersity index of 0.14 , an average diameter of $190.1 \pm 3 \mathrm{~nm}$, zeta potential of $-38.5 \mathrm{mV}$ and an entrapment efficiency of $73 \%$. The release profile of Benzocaine loaded in PLA nanocapsules showed a significant different behavior than that of the pure anesthetic in solution. This study is important to characterize a drug release system using benzocaine for application in pain treatment.

Keywords: local anesthesic; PLA nanocapsules; drug release.

\section{INTRODUÇÃO}

Anestésicos locais (AL) são compostos químicos cuja atividade farmacológica primária envolve o bloqueio reversível da condução nervosa. Essa propriedade é uma consequência da inibição do processo de excitação-condução, uma vez que interagem com canais de sódio voltagem-dependentes em nervos periféricos. Um AL exerce sua ação reversível em qualquer tecido nervoso podendo bloquear a passagem de impulsos tanto em fibras sensoriais quanto motoras aliviando ou evitando a dor, o que contribui para a grande utilização desses fármacos na prática médica. ${ }^{1-3}$

A benzocaína (Figura 1) é um AL do tipo éster que não apresenta na sua extremidade terminal um grupo amino como a maioria dos representantes dessa classe de fármacos. ${ }^{3}$ Sua ação é rápida e de curta duração, sendo usada principalmente em anestesia tópica. Benzocaína (BZC) é o agente anestésico tópico mais popular e, também, componente de várias formulações que são utilizadas clinicamente. Apesar de sua baixa toxicidade, a BZC não é totalmente inócua, uma vez que já foram descritos casos de aumento dos níveis de meta-hemoglobina após a administração de altas doses em crianças e alto potencial para provocar reações alérgicas ${ }^{4-8}$.

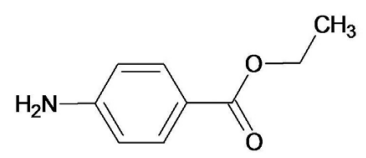

Figura 1. Estrutura química do anestésico local benzocaína

\footnotetext{
*e-mail: leonardo@sorocaba.unesp.br
}

Com o objetivo de aumentar a duração do efeito anestésico e reduzir a toxicidade de AL, tem-se utilizado como alternativa promissora a veiculação destes em sistemas de liberação modificada como, por exemplo, nanopartículas poliméricas. ${ }^{9-12}$ As nanopartículas poliméricas são sistemas carreadores de fármacos e outras moléculas que apresentam diâmetro inferior a $1 \mu \mathrm{m}$ e incluem as nanocápsulas (NC) e as nanoesferas (NE). Esses dois sistemas diferem entre si segundo a composição e organização estrutural. As NC são constituídas por um invólucro polimérico e um núcleo oleoso, podendo o fármaco estar dissolvido neste núcleo e/ou adsorvido à parede polimérica. Já as NE são formadas por uma matriz polimérica e não apresentam óleo em sua composição. Neste sistema, o fármaco pode ficar retido ou adsorvido. ${ }^{13,14}$

Trabalhos da literatura descrevem o desenvolvimento de formulações para liberação de benzocaína, dentre eles, destacam-se aqueles que usam lipossomas, ${ }^{7,15}$ géis ${ }^{15}$ e ciclodextrinas (CD). ${ }^{16}$

Mura e colaboradores ${ }^{15}$ descreveram o desenvolvimento e avaliação in vivo de uma formulação tópica lipossomal para contendo BZC, mostrando tanto a liberação sustentada quanto a potencialização do efeito anestésico em relação à formulação não-lipossomal.

Já Pinto e colaboradores ${ }^{16}$ relataram que a inclusão de BZC em $\beta$-CD aumentou a solubilidade desta de 4 para $16 \mathrm{mM}$ além de reduzir o potencial citotóxico da molécula de BZC.

No entanto, somente alguns trabalhos na literatura apresentam a associação de $\mathrm{AL}$ em sistemas poliméricos micro e nanoestruturados. ${ }^{9-11,17,18}$ Para a BZC, a associação com sistemas de micro e nanopartículas poliméricas não foi, até então, descrita na literatura. Neste trabalho, foram desenvolvidas nanocápsulas utilizando o polímero biodegradável poli (L-lactídeo) veiculando a BZC. Dentre as vantagens de se utiizar este sistema para a BZC destacam-se: a possibilidade de aumento de solubilidade (propriedade extremamente 
útil para a difusão da molécula através dos tecidos até atingir o local de ação); alteração no perfil de liberação deste fármaco e, redução das concentrações plasmáticas, bem como de efeitos tóxicos locais e sistêmicos, especialmente em relação a aqueles sistemas (lipossomas e CDs) já relatados na literatura. ${ }^{15,16}$

Este estudo teve como objetivo caracterizar a associação entre a BZC e as nanocápsulas de PLA através de medidas de tamanho (diâmetro hidrodinâmico) e potencial zeta (estabilidade coloidal), medidas de estabilidade química do polímero em função do tempo (análise de alterações de $\mathrm{pH}$ da suspensão), determinação da taxa de associação deste fármaco nas nanocápsulas de PLA e realização de ensaio de liberação in vitro a fim de verificar alterações no perfil de liberação do fármaco a partir do sistema carreador.

\section{PARTE EXPERIMENTAL}

\section{Materiais}

Foram utilizados benzocaína (Sigma Aldrich Chem. Co.), ácido poli-D,L-lactídeo (PLA) (Sigma Aldrich Chem. Co.), monoestearato de sorbitano $\left(\right.$ Span60 ${ }^{\circledR}$ ) (Sigma Aldrich Chem. Co.), polisorbato 80 (Tween80 ${ }^{\circledR}$ ) (LabSynth, Brasil), triglicerídeo de ácido caprílico/ cáprico (Miglyol 810 ${ }^{\circledR}$ ) (Hüls, Alemanha) e acetona grau analítico (LabSynth, Brasil). Os solventes empregados para análises cromatográficas foram acetonitrila grau HPLC (JT Baker ${ }^{\circledR}$ ) e água Milli-Q.

\section{Metodologia}

\section{Preparo das nanocápsulas de PLA com benzocaína}

O preparo das nanocápsulas de PLA foi realizado segundo o método de nanoprecipitação, envolvendo a mistura de uma fase orgânica em outra fase aquosa. A fase orgânica foi constituída do polímero PLA (100 mg), acetona (30 mL), benzocaína (40 mg), monestearato de sorbitano (40 mg) e triglicerídeo de ácido caprílico/capróico (200 mg). A fase aquosa foi composta por polissorbato 80 (60 mg) e água desionizada (30 mL). Após a dissolução dos componentes de ambas as fases, a fase orgânica foi adicionada lentamente sobre a fase aquosa com o auxílio de um funil pequeno. A suspensão resultante foi mantida sob agitação durante 10 min e, em seguida, foi concentrada sob baixa pressão até o volume de $10 \mathrm{~mL}$, com o auxílio de um evaporador rotativo, ${ }^{19}$ a fim de obter uma concentração de benzocaína de $4 \mathrm{mg} / \mathrm{mL}$. Foi também realizada uma formulação controle onde apenas a benzocaína foi eliminada da metodologia descrita acima.

\section{Medidas de tamanho e polidispersão}

A técnica de espalhamento dinâmico de luz foi utilizada para determinação do tamanho médio das partículas (diâmetro hidrodinâmico) e polidispersão. As análises foram realizadas diluindo-se (1/100) as suspensões de nanocápsulas (com e sem BZC) com água Milli-Q, utilizando-se um analisador de partículas Zeta plus ${ }^{\circledR}$ com detector em um ângulo fixo de $90^{\circ}$ e temperatura de $25^{\circ} \mathrm{C}$. As distribuições de tamanho e polidispersão foram medidas e expressas como média de cinco determinações..$^{9,12,20}$

\section{Medidas de potencial zeta}

$\mathrm{O}$ valor de potencial zeta, dado em $\mathrm{mV}$, foi determinado utilizando-se o analisador de potencial zeta, Zeta plus ${ }^{\circledR}$. As análises foram realizadas diluindo-se (1/100) as suspensões de nanocápsulas (com e sem BZC) em água Milli-Q, sendo os resultados médias de oito determinações.

Medidas de estabilidade química

A fim de verificar a estabilidade química da formulação foi realizada a medida de $\mathrm{pH}$ das suspensões de NC de PLA contendo BZC (eletrodo Corning, potenciômetro Tecnal) em função do tempo, uma vez que a alteração de $\mathrm{pH}$ pode indicar degradação do polímero. ${ }^{13}$

A medida dos valores de $\mathrm{pH}$ das suspensões de $\mathrm{NC}$ foi realizada durante um período de 60 dias, utilizando-se um potenciômetro calibrado com soluções tampão padrão em pH 7,0 e 4,0.

\section{Medidas de estabilidade coloidal}

Espectros ópticos na faixa de 200 a $600 \mathrm{~nm}$ (espectrofotômetro Cary-50, Varian, Inc.) foram coletados para as formulações de nanocápsulas de PLA com e sem o anestésico local em função do tempo. ${ }^{21,22}$ As suspensões de NC de PLA com e sem BZC foram analisadas, utilizando-se cubetas de quartzo com $1 \mathrm{~cm}$ de caminho óptico e diluindo-se as suspensões de NC de PLA com água desionizada $(1 / 100, v / v)$ para que as absorbâncias não ultrapassassem o valor de 1 (faixa linear, Lei de Beer).

\section{Medidas da taxa de associação da benzocaína nas nanocápsulas de PLA}

A porcentagem de BZC associada às nanocápsulas foi determinada pelo método de ultrafiltração/centrifugação. As amostras de nanocápsulas contendo benzocaína foram centrifugadas em filtros de ultrafiltração constituídos de celulose regenerada com poro de exclusão molecular de $30 \mathrm{kDa}$ (Microcon - Millipore ${ }^{\circledR}$ ), sendo o filtrado quantificado por cromatografia líquida de alta eficiência (CLAE). A taxa de associação da BZC foi determinada pela diferença entre a quantificação da concentração do fármaco no filtrado e a concentração total (100\%) do mesmo, presente na suspensão de nanocápsulas. ${ }^{13,23}$

As condições cromatográficas utilizadas para a quantificação foram: fase móvel composta por acetonitrila e água (50:50 v/v) em fluxo de $1 \mathrm{~mL} / \mathrm{min}$. A benzocaína foi detectada em comprimento de onda de $285 \mathrm{~nm}$ utilizando-se um detector de ultravioleta (UV). O volume de injeção foi de $20 \mu \mathrm{L}$ e todas as amostras injetadas foram filtradas previamente em membrana de polietersulfona de $0,22 \mu \mathrm{M}$ (Millipore $^{\circledR}$ ). A BZC total (100\%) presente na suspensão de nanocápsulas de PLA foi determinada diluindo-se a suspensão em acetonitrila. A acetonitrila consegue dissolver o polímero, liberando totalmente a BZC que, por sua vez, foi quantificada a partir da curva analítica validada segundo a Resolução RE no 899/2003 da Agência Nacional de Vigilância Sanitária (ANVISA).

\section{Ensaio de liberação in vitro da benzocaína}

A análise do perfil de liberação da BZC livre e associada às nanocápsulas foi realizada através de um sistema composto por dois compartimentos, sendo um compartimento doador $(2 \mathrm{~mL})$ e outro aceptor $(250 \mathrm{~mL}$, tampão Hepes, $\mathrm{pH} 7,4)$ separados por uma membrana de celulose com poros de exclusão molecular de $1000 \mathrm{Da}$, mantidos sob agitação. ${ }^{24} \mathrm{O}$ experimento foi realizado em condição de diluição sink.

Alíquotas de $2 \mathrm{~mL}$ foram retiradas do compartimento aceptor em intervalos de 15,30 e $60 \mathrm{~min}$, durante $330 \mathrm{~min}$ e quantificados por CLAE (utilizando condições analíticas descritas para a determinação da taxa de associação). Após cada alíquota retirada o volume do compartimento aceptor foi preenchido com tampão para que se mantivesse constante. As medidas das áreas dos cromatogramas obtidos foram convertidas em porcentagem de fármaco liberado utilizando como padrão uma solução da BZC livre em tampão.

Para análise do comportamento de liberação da BZC a partir das nanocápsulas de PLA, foi utilizado o modelo teórico descrito por Korsmeyer-Peppas para liberação de fármacos de sistemas poliméricos. ${ }^{25} \mathrm{O}$ modelo de Korsmeyer-Peppas leva em consideração que o 
mecanismo de liberação do fármaco não segue a lei de Fick e segue um comportamento anômalo descrito pela Equação 1.

$M / M_{\infty}=k t^{n}$

onde $M_{t}$ é a quantidade de fármaco liberada em um determinado tempo $t, M_{\infty}$ é a quantidade de fármaco liberada em um tempo infinito, $k$ é a constante cinética de liberação e $n$ é o expoente de liberação. $\mathrm{O}$ valor de $n$ é relacionado com a forma geométrica do sistema de liberação e determina o mecanismo de liberação; $n$ é igual a 0,45 para processos controlados por difusão, enquanto valores de $n$ entre 0,45 e 1,0 indicam um transporte anômalo não Fickniano.$^{26} \mathrm{O}$ modelo de Kornmeyer-Peppas é geralmente usado para analisar a liberação de um fármaco de uma matriz polimérica quando este não é bem conhecido ou quando um ou mais tipos de fenômenos estão envolvidos. A linearização da Equação 1 através da construção do gráfico de $\ln M_{t} / M_{\infty}$ em função de $\ln t$, fornece o expoente de liberação $(n)$ e a constante de liberação $\left(k\right.$, tempo $\left.^{-n}\right){ }^{26}$

\section{RESULTADOS E DISCUSSÃO}

As medidas de tamanho (diâmetro hidrodinâmico), polidispersão e potencial zeta das partículas são parâmetros indicativos de estabilidade das nanocápsulas em suspensão. A polidispersão indica a distribuição de tamanho das nanocápsulas e, normalmente, valores menores que 0,2 para suspensões coloidais são considerados bons indicadores de estabilidade. ${ }^{27}$ Já o potencial zeta reflete a carga de superfície das nanocápsulas, sendo que este parâmetro pode ser influenciado pela composição da partícula, meio dispersante, $\mathrm{pH}$ e força iônica presentes na solução. Normalmente, nanopartículas (dentre elas as nanocápsulas) que apresentam valores de potencial zeta próximos $\mathrm{a} \pm 30 \mathrm{mV}$ possuem boa estabilidade coloidal em solução. ${ }^{27}$

A Tabela 1 apresenta os valores medidos para os parâmetros descritos acima para as suspensões de NC de PLA com e sem BZC.

Tabela 1. Valores de diâmetro médio (diâmetro hidrodinâmico, nm), polidispersão e potencial zeta $(\mathrm{mV})$ das suspensões de NC de PLA com e sem BZC

\begin{tabular}{lll}
\hline Parâmetro & \multicolumn{1}{c}{ NC-PLA } & BZC:NC-PLA \\
\hline Diâmetro médio $(\mathrm{nm})$ & $205,6 \pm 05$ & $190,1 \pm 03$ \\
Polidispersão & $0,145 \pm 0,020$ & $0,135 \pm 0,010$ \\
Potencial Zeta $(\mathrm{mV})$ & $-33,5 \pm 0,8$ & $-38,5 \pm 0,7$ \\
\hline
\end{tabular}

Os resultados apresentados na Tabela 1 mostram que as $\mathrm{NC}$ de PLA com ou sem BZC apresentam tamanho (diâmetro hidrodinâmico) e polidispersão compatíveis com suspensões coloidais, mostrando que o fármaco não afeta de maneira significativa estes parâmetros. ${ }^{28}$ Os valores de potencial zeta destas formulações indicam para uma boa estabilidade em solução, uma vez que os valores encontrados são maiores, em módulo, que $-30 \mathrm{mV} .^{28}$

A fim de observar a estabilidade química do polímero presente nas nanocápsulas em função do tempo, foi acompanhado o valor de pH da suspensão (Figura 2). Esta análise é importante para assegurar a estabilidade química do polímero presente nas nanocápsulas, pois a alteração de $\mathrm{pH}$ pode indicar degradação do polímero em solução, gerando monômeros (no caso ácido láctico) que levam à acidificação da formulação. ${ }^{12}$

A partir dos resultados apresentados na Figura 2 pode-se observar que o valor de $\mathrm{pH}$ inicial da formulação de NC-PLA contendo $\mathrm{BZC}$ foi de 3,74, valor próximo ao descrito na literatura para $\mathrm{NC}$ de PLA. ${ }^{28}$ Além disso, observou-se um aumento no $\mathrm{pH}$ da formulação

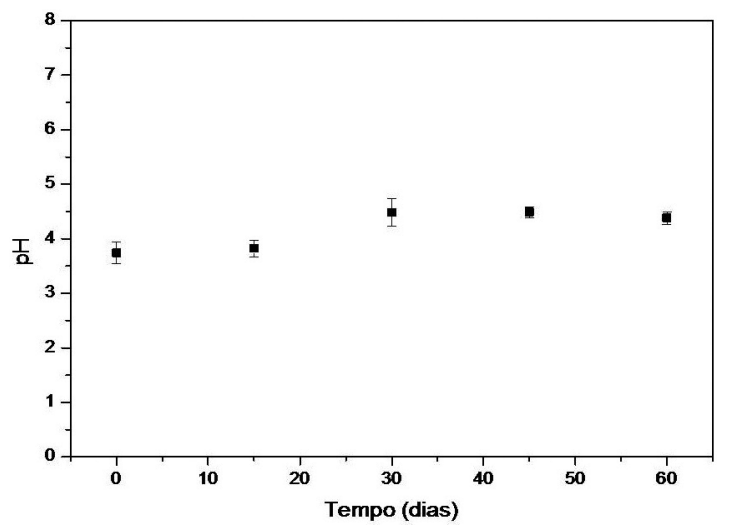

Figura 2. Variação de $\mathrm{pH}$ da formulação de nanocápsulas de PLA com BZC no período compreendido entre zero e 60 dias de armazenamento em temperatura ambiente $(n=3)$

em função do tempo de análise sendo de 3,74, inicialmente, e de 4,38 em 60 dias após a preparação. $\mathrm{O}$ aumento do valor de $\mathrm{pH}$ medido para as NC-PLA contendo BZC após estocagem a temperatura ambiente pode ser explicado pela agregação do polímero com concomitante liberação da fase oleosa no meio. ${ }^{29}$

Além do acompanhamento da estabilidade química do polímero (através da medida de valores de $\mathrm{pH}$ ) foi também estudada a estabilidade coloidal das suspensões de nanocápsulas de PLA contendo BZC, através da análise em função do tempo de espectros ópticos das formulações (Figura 3).

A análise do espectro de absorção fornece informações referentes ao espalhamento de luz provocado pelas nanocápsulas presentes nas amostras e, por consequência, quanto maior o tamanho das partículas em solução maior a dispersão de luz. ${ }^{21,22}$ Sendo assim, caso ocorra agregação das partículas em solução em função do tempo, serão observadas alterações (aumento de absorbância) no espectro de absorção óptico das suspensões.

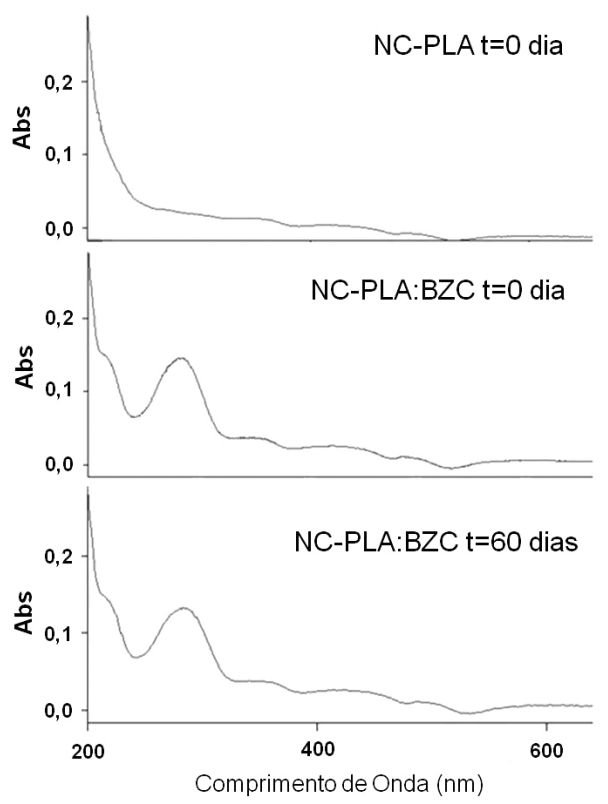

Figura 3. Espectro de absorção para formulações de nanocápsulas de PLA com e sem benzocaína em função do tempo (zero e 60 dias)

A Figura 3 apresenta o espectro de absorção óptico da formulação de nanocápsulas de PLA e PLA contendo BZC. Para as amostras de PLA sem BZC observa-se que ocorre uma maior absorção de luz 
na faixa próxima a $200 \mathrm{~nm}$. Já para as suspensões contendo BZC observa-se um pico de absorção máximo em $284 \mathrm{~nm}$, correspondente à absorção da molécula de BZC. ${ }^{7}$ Comparando-se o espectro de absorção das suspensões de nanocápsulas de PLA contendo BZC em tempo zero e após 60 dias não se observam alterações significativas nos dois espectros, indicando assim que as partículas em solução apresentam boa estabilidade coloidal. Trabalhos na literatura ${ }^{21,22}$ mostram que a técnica de espectrofotometria pode ser utilizada para medidas de cinética de agregação de partículas em solução, bem como alterações na absorção destas em função do tamanho.

Uma vez caracterizada a estabilidade química $(\mathrm{pH})$ e coloidal (espectros de absorção) das suspensões de nanocápsulas de PLA, foi determinado o teor de BZC nas nanocápsulas utilizando-se, para isso, uma curva analítica previamente validada para a BZC (área do pico cromatográfico $=0,09082+0,14085[\mathrm{BZC}], \mathrm{r}=0,9978$, limite de detecção $=3,92 \mu \mathrm{M}$ e limite de quantificação $=13,6 \mu \mathrm{M}$ ).

Neste trabalho, a partir dos dados de concentração total de BZC presente na suspensão de nanocápsulas (100\%) e dos dados de concentração de BZC não associada às nanocápsulas foi possível calcular a taxa de associação, sendo o valor encontrado de $73 \%$.

$\mathrm{O}$ valor de associação das moléculas de BZC nas nanocápsulas de PLA é devido a este fármaco apresentar uma baixa solubilidade aquosa, o que faz com que a interação com a matriz polimérica e bem como com o interior oleoso das nanocápsulas seja alto.

Nesta formulação a quantidade de BZC utilizada nas suspensões de nanocápsulas de PLA foi de $4 \mathrm{mg} / \mathrm{mL}$, ou seja, bem acima do valor de solubilidade aquosa da benzocaína $(0,66 \mathrm{mg} / \mathrm{mL}){ }^{7} \mathrm{~A}$ associação da BZC à formulação de nanocápsulas de PLA levou a um aumento de solubilidade de seis vezes, fato esse que já é suficiente para se inferir um aumento no efeito anestésico, uma vez que um dos fatores que limitam a utilização da BZC por via infiltrativa é a sua baixa solubilidade aquosa. ${ }^{7}$

A partir dos ensaios de liberação in vitro foi possível observar o efeito da associação da BZC nas nanocápsulas de PLA no perfil de liberação deste anestésico. Neste ensaio, o fármaco possui a capacidade de atravessar os poros da membrana, porém as nanocápsulas não a atravessam e, desta forma, é possível observar o efeito da associação na velocidade de liberação da molécula de BZC. A Figura 4 apresenta as curvas de porcentagem de liberação para a molécula de BZC livre e associada às nanocápsulas em função do tempo (330 min).

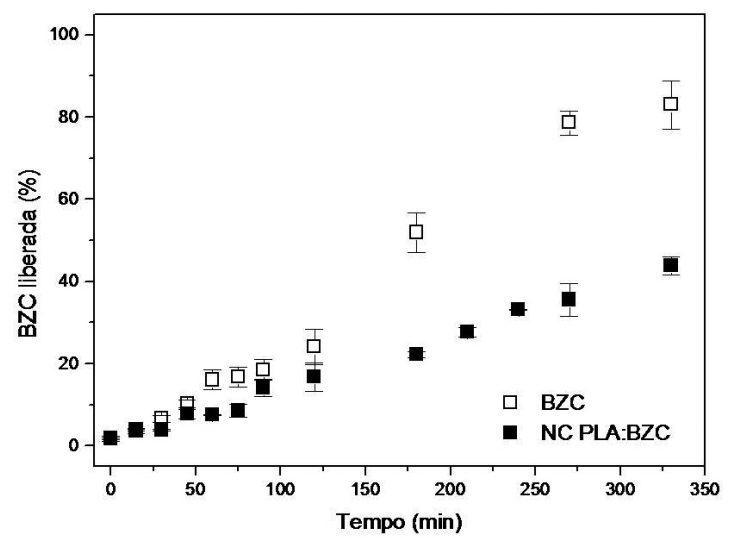

Figura 4. Liberação cumulativa da BZC (\%) em solução e em suspensão de nanocápsulas de PLA $(n=3)$

Pela análise das curvas se observa que no tempo de 330 min a liberação da BZC livre foi de $85 \%$, enquanto que para a BZC associada às nanocápsulas de PLA a liberação foi de $45 \%$. Sendo assim, a associação de BZC com nanocápsulas modifica o perfil de liberação deste fármaco quando comparada à benzocaína sozinha. A menor taxa de liberação da BZC neste caso deve-se a estas moléculas estarem associadas ao núcleo oleoso e invólucro polimérico das nanocápsulas, o que faz com que a difusão da molécula para o compartimento aceptor seja mais lento. ${ }^{30}$

Neste sentido, sabe-se que o mecanismo para a liberação de fármacos de nanopartículas (como as nanocápsulas) é determinado por diferentes fenômenos físico-químicos ${ }^{31} \mathrm{O}$ modelo de KorsmeyerPeppas (Equação 1), um modelo semi-empírico que correlaciona a liberação do fármaco em função do tempo por uma equação exponencial simples e tem sido usado para avaliar a liberação de fármacos de dispositivos poliméricos de liberação controlada, especialmente quando o mecanismo de liberação do fármaco não é conhecido ou quando existe mais que um mecanismo envolvido. ${ }^{25,26,31}$ Sistemas de liberação porosos podem apresentar valores de $n<0,45$, sendo este devido à combinação de processos de difusão através da matriz polimérica e difusão parcial através de poros preenchidos com água no interior da matriz polimérica. ${ }^{26}$

A fim de determinar se o mecanismo de liberação da BZC a partir das nanocápsulas de PLA é controlado por difusão de Fick, o resultado da curva de liberação foi tratado pelo modelo de Korsmeyer-Peppas. Quando a equação do modelo de Korsmeyer-Peppas é convertida para logaritmo, a equação se torna $\ln M / M=\ln k+n \ln t$ e a inclinação $n$ pode ser determinada pelo gráfico do logaritmo da taxa de liberação em função do logaritmo do tempo.

De acordo com esse modelo, uma reta foi obtida pelo gráfico de liberação da BZC das nanocápsulas de PLA (Figura 5). O resultado da regressão linear mostra um valor de expoente de liberação $n=$ $0,95(\mathrm{r}=0,989)$ e $0,0016 \mathrm{~min}^{-\mathrm{n}}$ para a constante da taxa de liberação (k). O resultado do valor de $n$ mostra que a BZC é liberada devido a transporte anômalo não governado por difusão de Fick. ${ }^{26,31}$

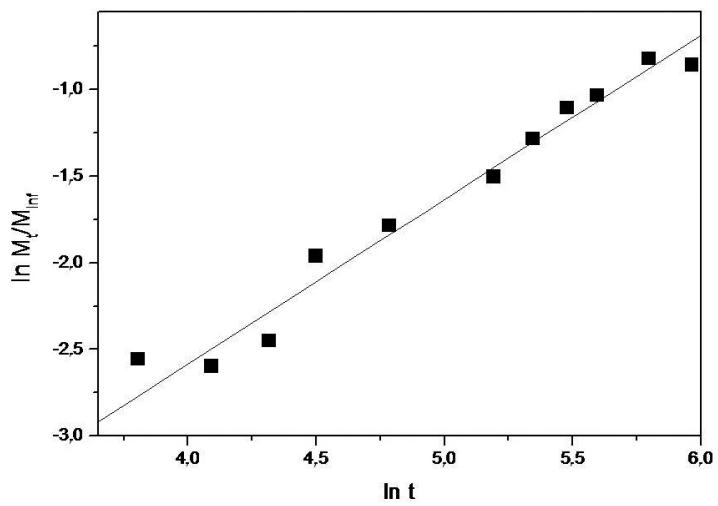

Figura 5. Gráfico de ln $M / M_{¥}$ em função do $\ln t$, de acordo com o modelo de Korsmeyer-Peppas $(n=3)$

\section{CONCLUSÃO}

Os resultados indicam que as nanocápsulas de PLA contendo BZC apresentam boa estabilidade química (pequena variação de $\mathrm{pH}$ ) e coloidal (sem alterações no espectro de absorção de luz das formulações) durante um período de 60 dias. A taxa de associação da BZC nas nanocápsulas de PLA de $73 \%$ modificou o perfil de liberação da benzocaína, fazendo com que a solubilidade em água da BZC fosse aumentada em 6 vezes. A análise do perfil de liberação utilizando o modelo de Korsmeyer-Peppas mostrou que a BZC é liberada das nanocápusulas de PLA por um processo de transporte anômalo não governado por difusão de Fick. Este estudo fornece subsídios para avaliações in vivo quanto ao uso da nova formulação de benzocaína em nanocápsulas por via tópica e, especialmente, também indica perspectivas de possíveis estudos envolvendo a administração dessa formulação por via infiltrativa. 


\section{AGRADECIMENTOS}

À FAPESP (06-00121-9, 07/00127-0), CNPq e Fundunesp pelos auxílios financeiros. N. F. S. de Melo e R. Grillo agradecem as bolsas de iniciação científica da Fapesp. Os autores agradecem também as contribuições dos assessores da Química Nova.

\section{REFERÊNCIAS}

1. Covino, B. G.; Vassalo H. G.; Anestésicos locais: mecanismos de ação e uso clínico, Colina: Rio de Janeiro, 1985.

2. Fraceto, L. F.; Oyama, S. Jr.; Nakaie, C. R.; Spisni, A.; de Paula, E.: Pertinhez, T. A.; Biophys. Chem. 2006, 20, 29; Malheiros, S. V. P.; Pinto, L. M. A.; Gottardo, L.; Yokaichiya, D. K.; Fraceto, L. F.; Meirelles, N. C.; de Paula, E.; Biophys. Chem. 2004, 110, 213; de Paula, E.; Schreier, S.; Jarrel, H. C.; Fraceto, L. F.; Biophys. Chem. 2008, 132, 47.

3. de Araújo, D. R.; de Paula, E.; Fraceto, L. F.; Quim. Nova 2008, 31, 1775 .

4. Yagiela, J. A.; Neidle, E. A.; Dowd, F. J.; Farmacologia e terapêutica para dentistas, 4ª ed., Guanabara Koogan: Rio de Janeiro, 1998.

5. Bennett, R. C.; Monheim: Anestesiologia local e controle da dor na prática dentária, $7^{\mathrm{a}}$ ed., Guanabara Koogan: Rio de Janeiro, 1989.

6. Ellis, B. F.; Seiler, J. G.; Palmore, M. M.; J. Bone Joint Surg. Am. 1995, $77,937$.

7. Pinto, L. M. A.; Yokaichiya, D. K.; Fraceto, L. F.; de Paula, E.; Biophys. Chem. 2000, 87, 213.

8. Balicer, R. D.; Kitai, E.; TheScientificWorldJournal 2004, 4, 517.

9. Gorner, T.; Gref, R.; Michenot, D.; Sommer, F.; Tran, M. N.; Dellacherie, E.; J. Control. Release 1999, 57, 259.

10. Govender, T.; Stolnik, S.; Garnett, M. C.;Illum, L.; Davis, S. S.; J. Control. Release 1999, 57,171; Moraes, C. M.; de Paula, E.; Rosa, A H.; Fraceto, L. F.; Quim. Nova 2008, 31, 2152; Moraes, C. M.; Matos, A. P.; Lima, R.; Rosa, A. H.; de Paula, E.; Fraceto, L. F.; J. Biol. Phys. 2007, 33, 455 .

11. Polakovic, M.; Gorner, T.; Gref, R.; Dellacherie, E.; J. Control. Release 1999, 60,169.
12. Govender, T.; Riley, T.; Ehtezazi, T.; Garnett, M. C.; Stolnik, S.; Illum, L.; Davis, S. S.; Int. J. Pharm. 2000, 199, 95.

13. Schaffazick, S. R.; Guterres S. S.; Freitas, L. L.; Pohlmann, A. R.; Quim. Nova 2003, 26, 726 .

14. Durán, N.; Mattoso, L. H. C.; Morais, P. C.; Nanotecnologia, Artliber: São Paulo, 2006.

15. Mura, P.; Maestrelli, F.; González-Rodrýguez, M. L.; Michelacci, I.; Ghelardini, C.; Rabasco, A. M.; Eur. J. Pharm. Biopharm. 2007, 67, 86.

16. Pinto, L. M.; Fraceto, L. F.; Santana, M. H.; Pertinhez, T. A.; Junior, S. O.; de Paula, E.; J. Pharm. Biomed. Anal. 2005, 39, 956.

17. Blanco, M. D.; Bernardo, M. V.; Sastre, R. L. ; Olmo, R.; Muniz, E.; Teijón, J. M.; Eur. J. Pharm. Biopharm. 2003, 55, 229.

18. Klose, D.; Siepmann, F.; Elkharraz, K.; Krenzlin, S.; Siepmann, J.; Int. J. Pharm. 2006, 314, 198.

19. Fessi, H.; Puiseiux, F.; Devissaguet, J-P.; European Patent, 0274961 A1, 1988.

20. Venkatraman, S. S.; Jie, P.; Min, F.; Freddy, B. Y, C.; Leong-Huat, G. ; Int. J. Pharm. 2005, 298, 219.

21. Anal, A. K.; Tobiassen, A.; Flanagan, J.; Singh, H.; Colloids Surf., B 2008, 64, 104.

22. Guarino, A. J.; Lee, S. P.; Tulenko, T. N.; Wrenn, S. P.; J. Colloid Interface Sci. 2004, 279, 109.

23. Gamisans, F.; Lacoulonche, F.; Chauvet, A.; Espina, M.; Garcia, M. L.; Egea, M. A.; Int. J. Pharm. 1999, 179, 37.

24. Paavola, A.; Yliruusi, J.; Kalso, E.; Wahlström, T.; Rosenberg, P.; Pharm. Res. 1995, 12, 1997.

25. Korsmeyer, R. W.; Gurny, R.; Doelker, E. M.; Buri, P.; Peppas, N. A.; Int. J. Pharm. 1983, 15, 25.

26. Peppas, N. A.; Pharm. Acta Helv. 1985, 60,110.

27. Mohanraj, V. J.; Chen, Y.; Trop. J. Pharm. Res. 2006, 5, 561.

28. Guterres, S. S.; Fessi, H.; Barrat, G.; Devissaguet, J. P.; Puisieux, F.; Int. J. Pharm. 1995, 113, 57.

29. Pohlmann, A. R.; Weiss, V.; Mertins, O.; Silveira, N. P.; Guterres, S. S.; Eur. J. Pharm. Sci. 2002, 16, 305.

30. Romero-Cano, M. S.; Vicent, B. ; J. Control. Release 2002, 82, 127.

31. Costa, P.; Lobo, J. M. S.; Eur. J. Pharm. Sci. 2001, 13, 123. 